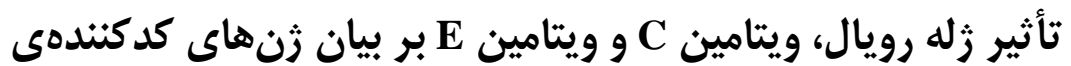

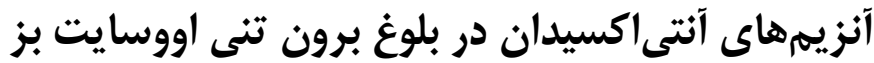

\author{
سعيد حيدرى'، حميد دلدار 'و زربخت انصارى بييرسر ايیى \\ ا و ץ- دانش آموخته كارشناسى ارشد و دانشيار، گروه علوم دامى، دانشگاه علوم كشاورزى و منابع طبيعى سارى

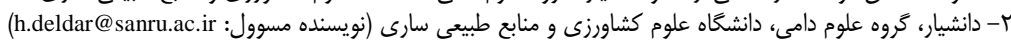

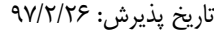 \\ تاريخ دريافت: دانثاه عام كثاوري
}

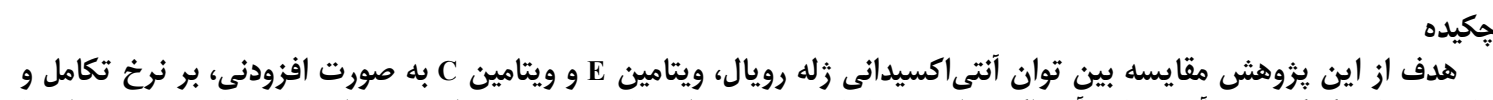

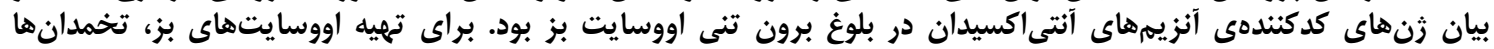

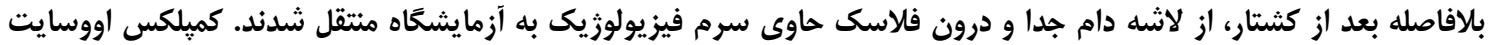

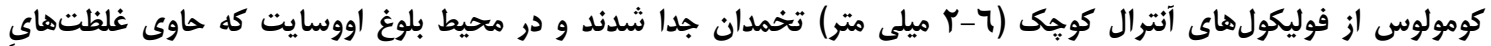

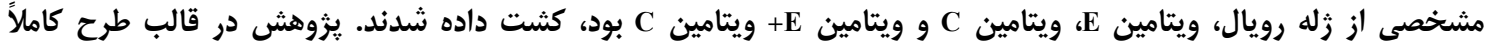

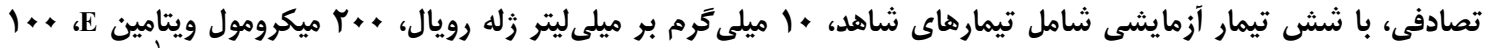

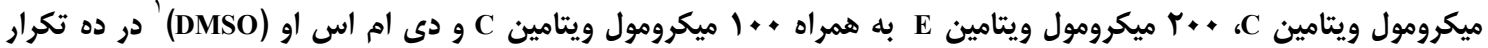

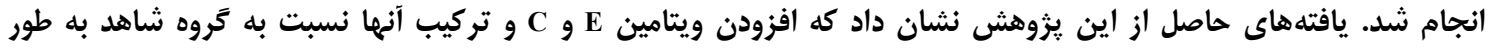

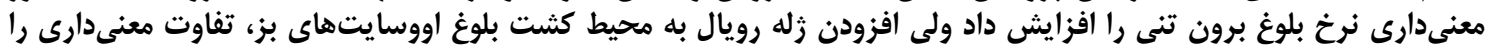

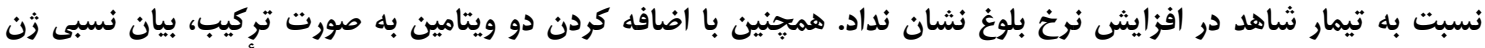

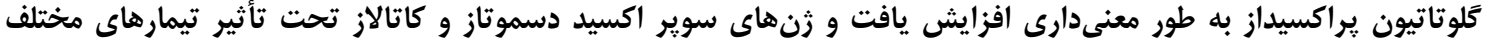

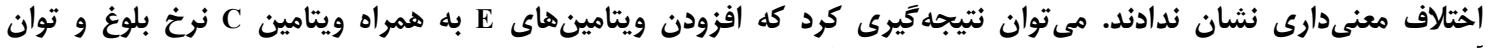
آنتىاكسيدانى اووسايت را نسبت تيمار زله رويال بيشتر افزايش دائ دادي

وازمهاى كليدى: زله رويال، ويتامين E، ويتامين C، بلوغ برون نتى، اووسايت بز

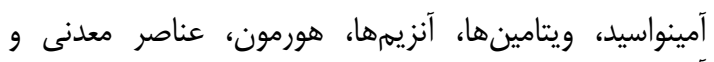

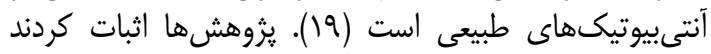

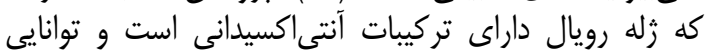

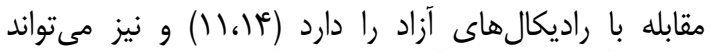

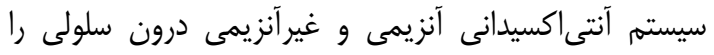

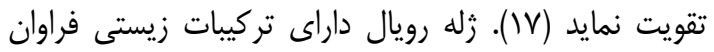

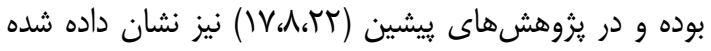

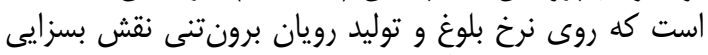

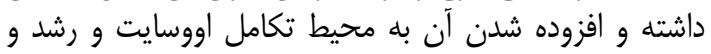

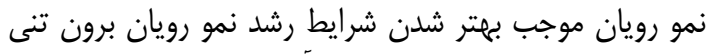

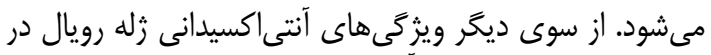

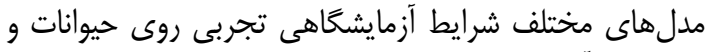

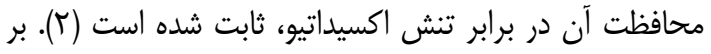

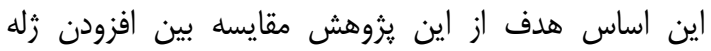

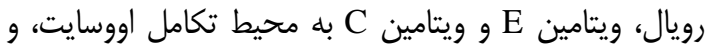

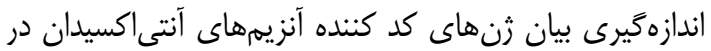
بلوغ برونتى اووسايت بز بود.

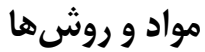

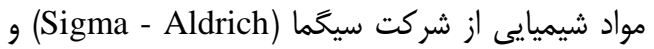
Gibco

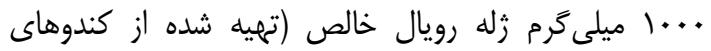

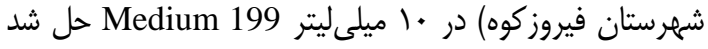

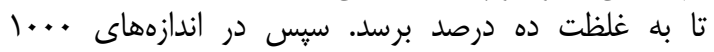

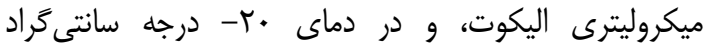
نكَدارى شد. براى بلوغ برونتنى اووسايتها نياز به اووسايت

1- Dimethyl Sulfoxide

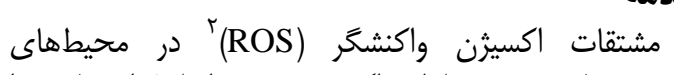

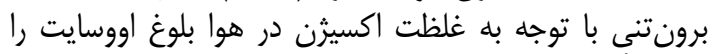

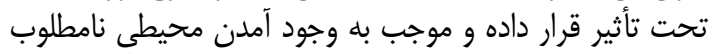

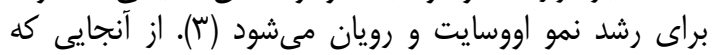

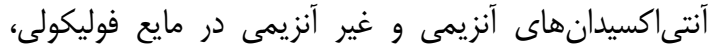

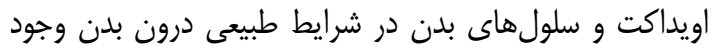

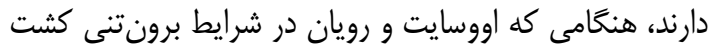

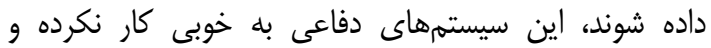

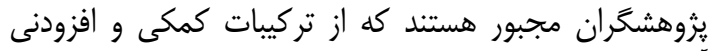

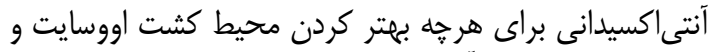

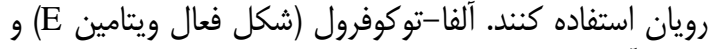

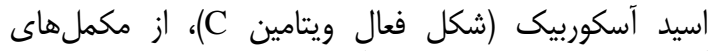

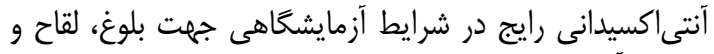

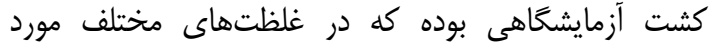
استفاده قرار مى كيرند. آلفا-توكوفرول يك يك آنتىاكسيدان

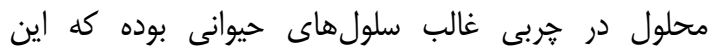

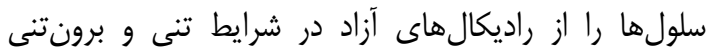

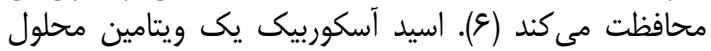

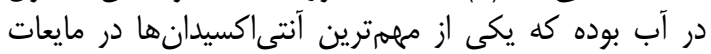

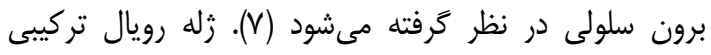

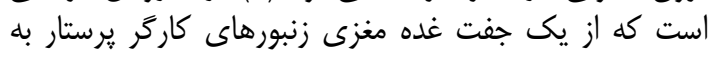

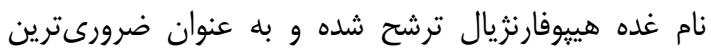

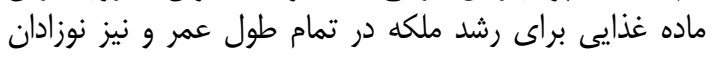

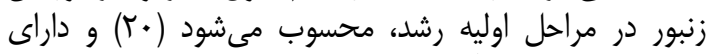

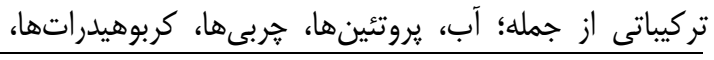




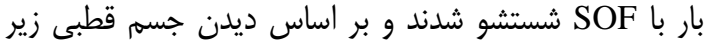

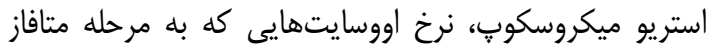

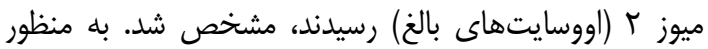

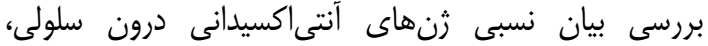

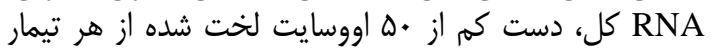

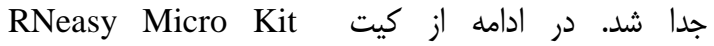
براى جداسازى RNA (QIAGEN, 74004)

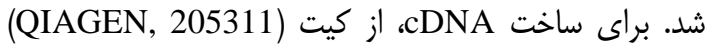
Quanti Tec Reves Transcription

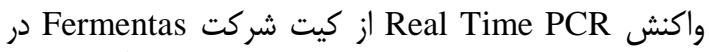
حجمهاى هاك الميكروليترى و و با استفاده از آغازكرهاى

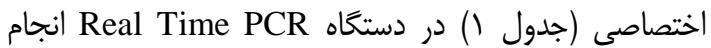

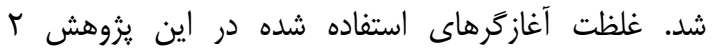

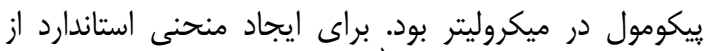

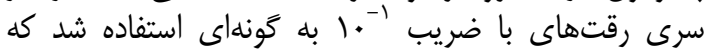

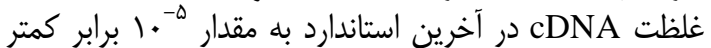

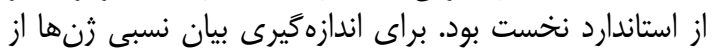

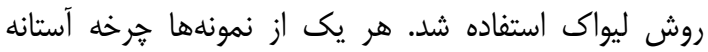

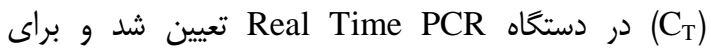

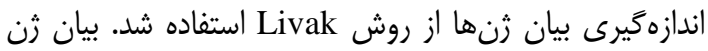

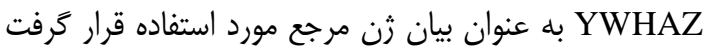

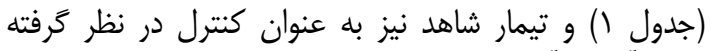

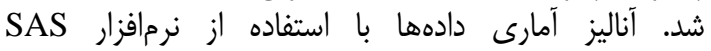

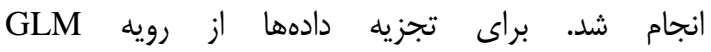
استفاده شد. هنگامى كه آناليز دادها معنىدار شد، آزمون دانكن براى مقار مايسه ميانكين

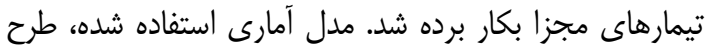

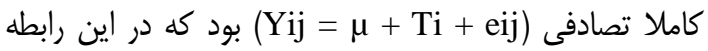

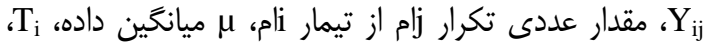

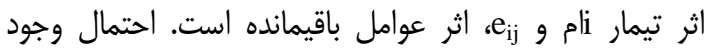

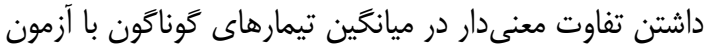
دانكن كوجگتر مساوى با ه درصد در نظر كرفته شد.

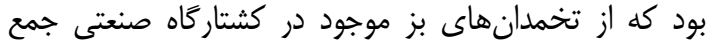

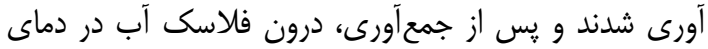

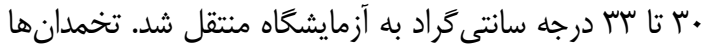

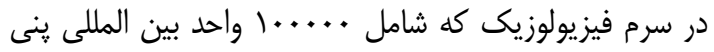

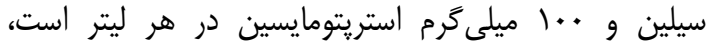

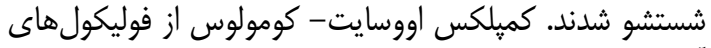

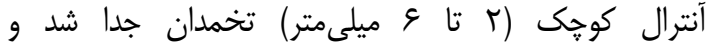

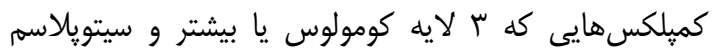

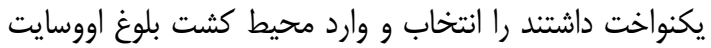

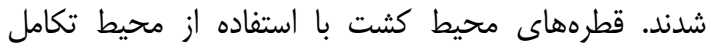

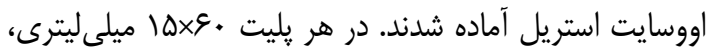

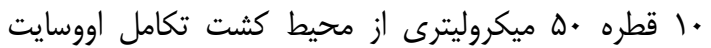

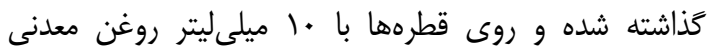

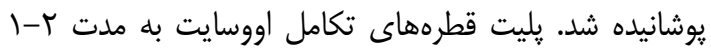

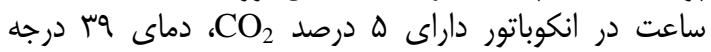

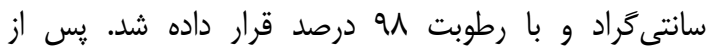
شستشوى كميلكس هاى اووسايت- كومولوس دران در محلول

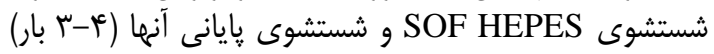

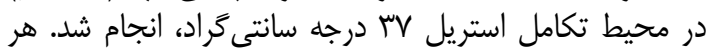

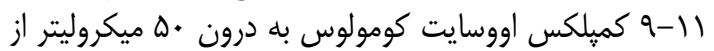

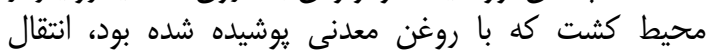

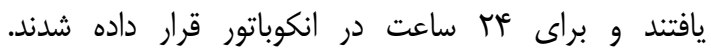

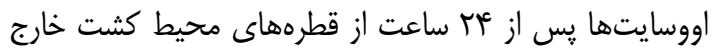

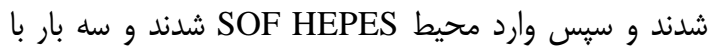

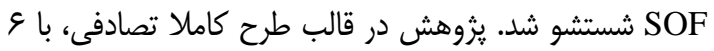

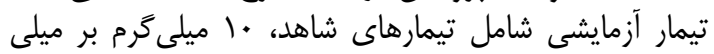

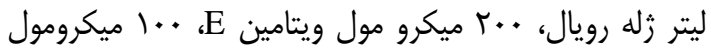

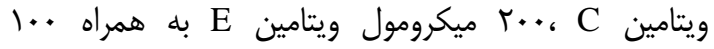

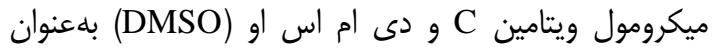

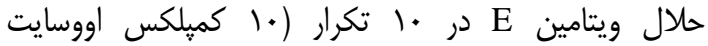

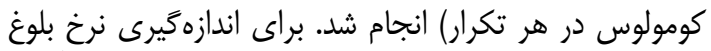

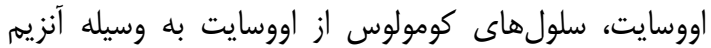
هيالورونيداز جدا شده و اووسايتهاى بدون كومولوس لئ نيز

Table 1. The seqvences primers seed for relative gene of expression

جدول ا- توالى آغازگرهاى استفاده شده براى بيان نسبى ثنها

\begin{tabular}{|c|c|c|c|}
\hline جفت باز & شماره شناسايى & آغازگ رفت و برگشتت & ثن \\
\hline ar & AB201469.1 & $\begin{array}{l}\text { F:5'-CAC TTC GAG GCA AAG GGA GA-3' } \\
\text { R:5'-CCA AAC TGA TGG ACG TGG AA-3' }\end{array}$ & SOD \\
\hline $1 \cdot v$ & GU131344.1 & $\begin{array}{l}\text { F:5-GGA TGA AAG TCC AGC CCA AG-3, } \\
\text { R:5'-GAC CAT ACC GCT TCA CCA CA-3' }\end{array}$ & GPX \\
\hline IVV & GQ204786 & $\begin{array}{l}\text { F:5'-CAA TGT TCT GAC GGT AGG GC-3' } \\
\text { R:5'-TTC GCC TTG GAG TAT CTG GT-3' }\end{array}$ & CAT \\
\hline 110 & AY970970 & $\begin{array}{l}\text { F:-5'-TGT AGG AGC CCG TAG GTCATCT-3' } \\
\text { R:5CTCTCTGTATTCTCGAGCCATCT-3' }\end{array}$ & YWHAZ \\
\hline
\end{tabular}

SOD: superoxide dismutase; GPX: gluthation peroxidase; CAT: catalase; YWHAZ: tyrosine 3-monooxygenase/tryptophan 5-monooxygenase activation protein zeta

ه D

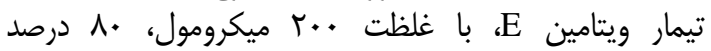

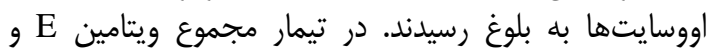

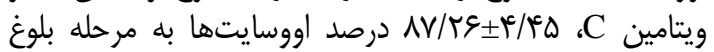

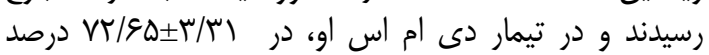

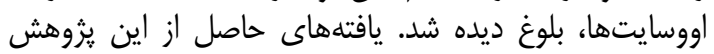

\section{نتايج و بحث}

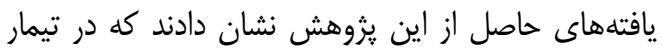
شاهد

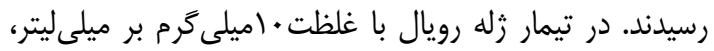

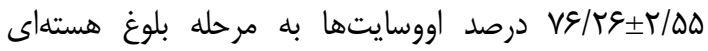

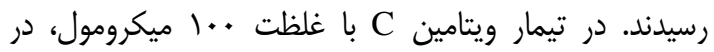




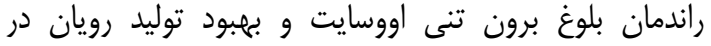

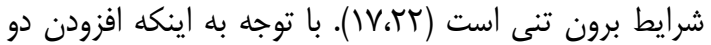

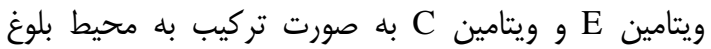

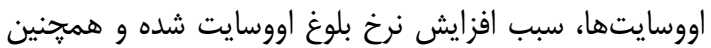

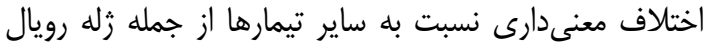

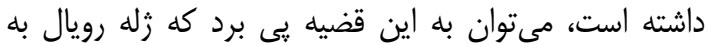

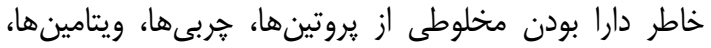

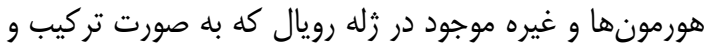

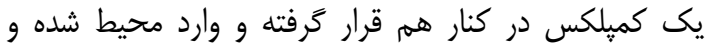

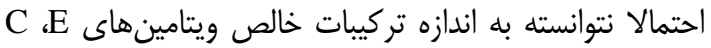

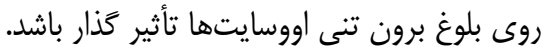

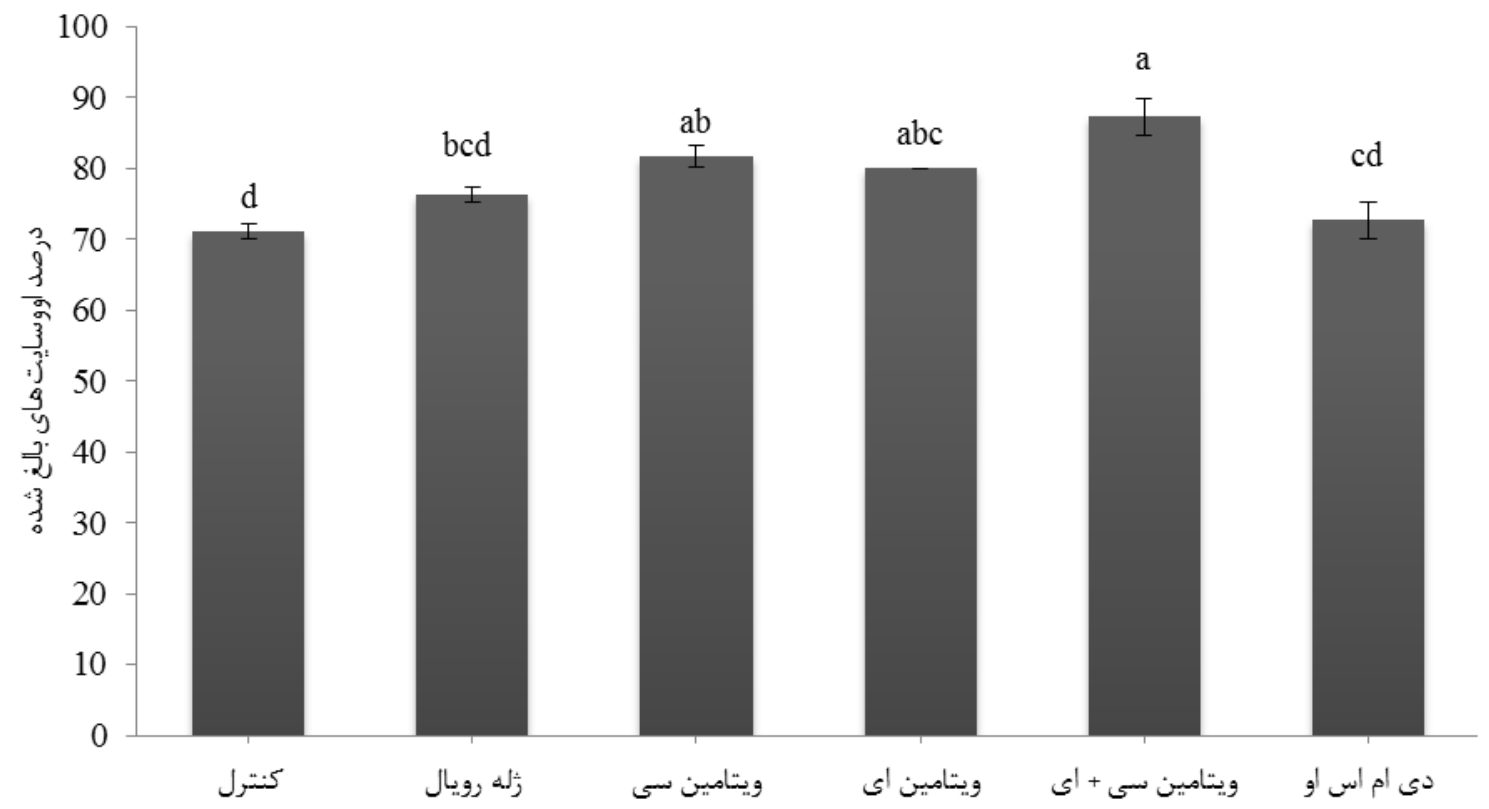

شكل (- تأثير افزودن ويتامينهاى E C C و زله رويال بر بلوغ برون تنى اووسايت بز

Figur 1. Effect of vitamin E, C and royal jelly on in vitro maturation of goat oocyte

سبب افزايش نرخ بلوغ و همجنين افزايش بيان برخى از

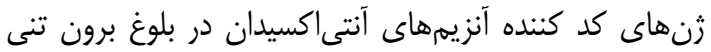

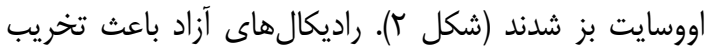

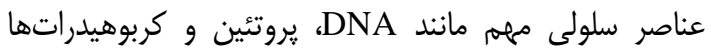

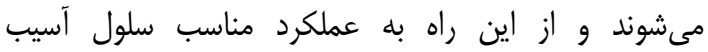

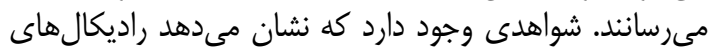

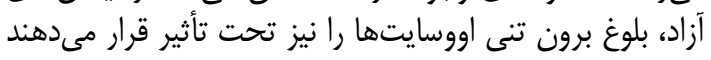

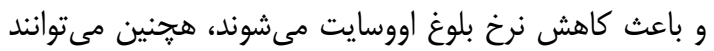

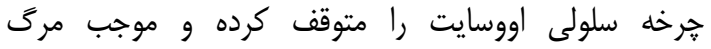

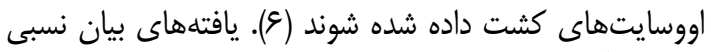

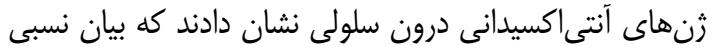

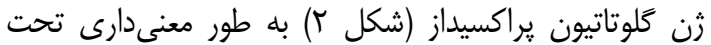

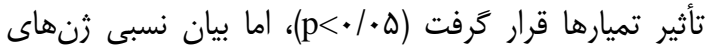

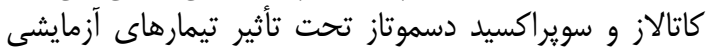

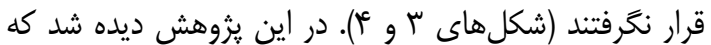

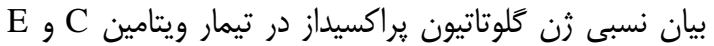

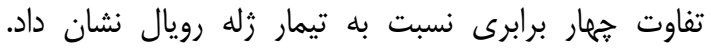

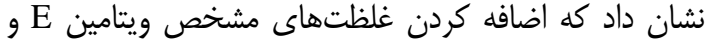

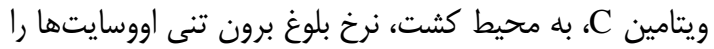

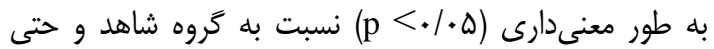

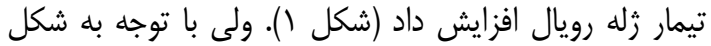

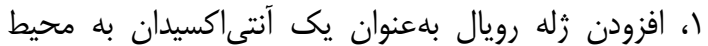

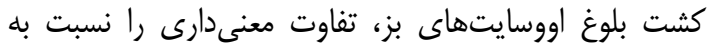

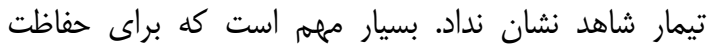

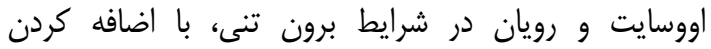

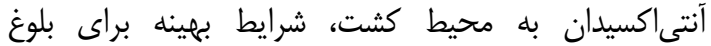

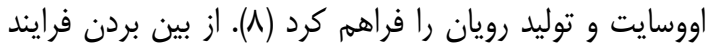
اكسيداسيون و يا كاهش آن يكى از عوامل مؤثر افزايش إن فراين 
عملكرد رشدى اووسايت مورد نياز هستند و نيز در كاهش

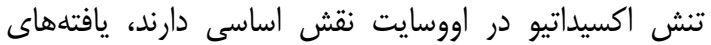

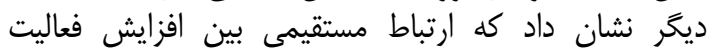

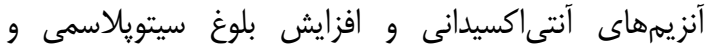
هستهاى اووسايت نيز وجود دارد (ع).

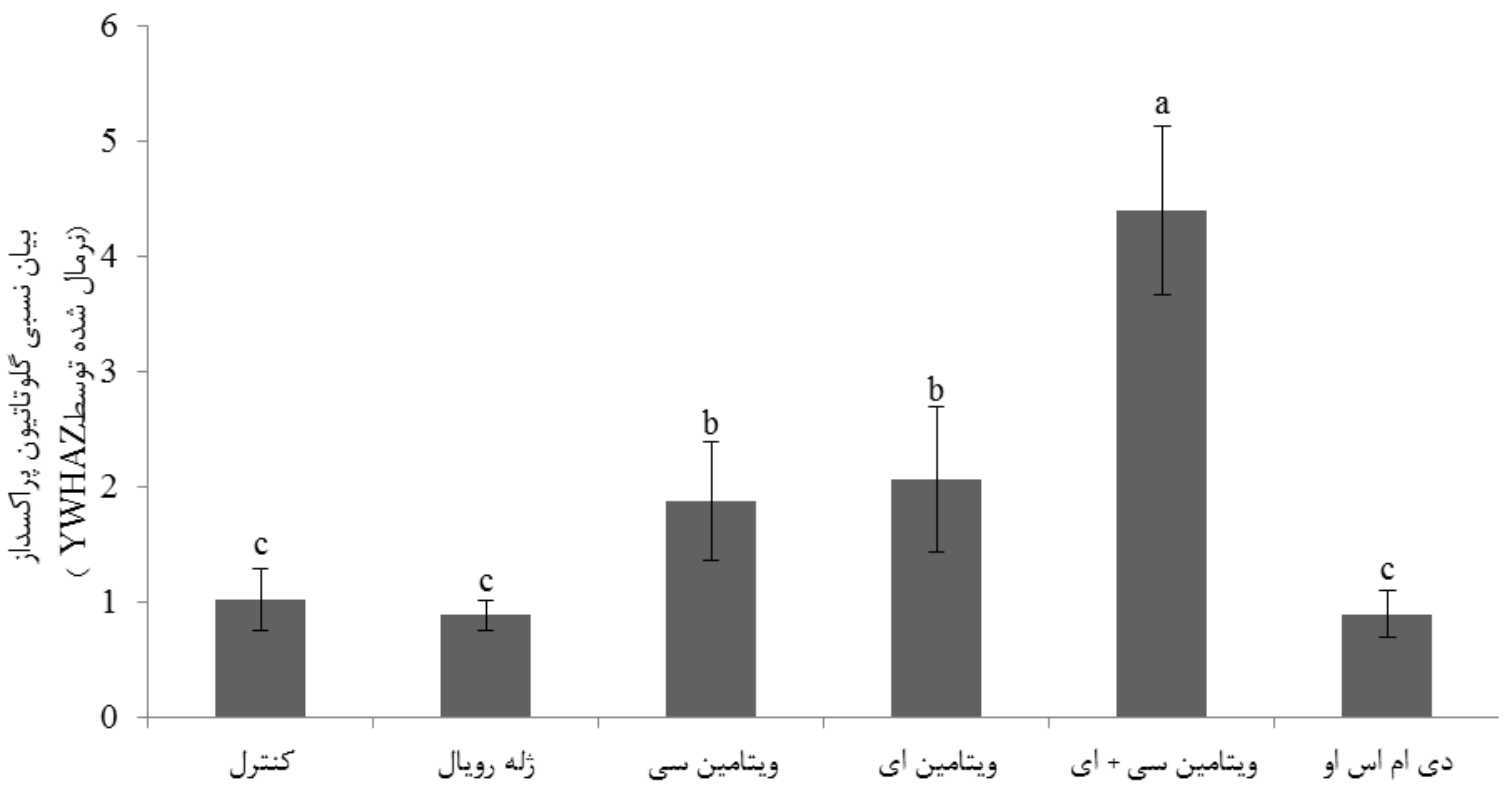

شكل r- تأثير افزودن ويتامينهاى E، C و زله رويال بر بيان نسبى ثن گلوتاتيون پراكسيداز در اووسايت بز

Figure 2. Effect of vitamin E, C and royal jelly on gluthation prtoxidase relative gene expression in goat oocyte

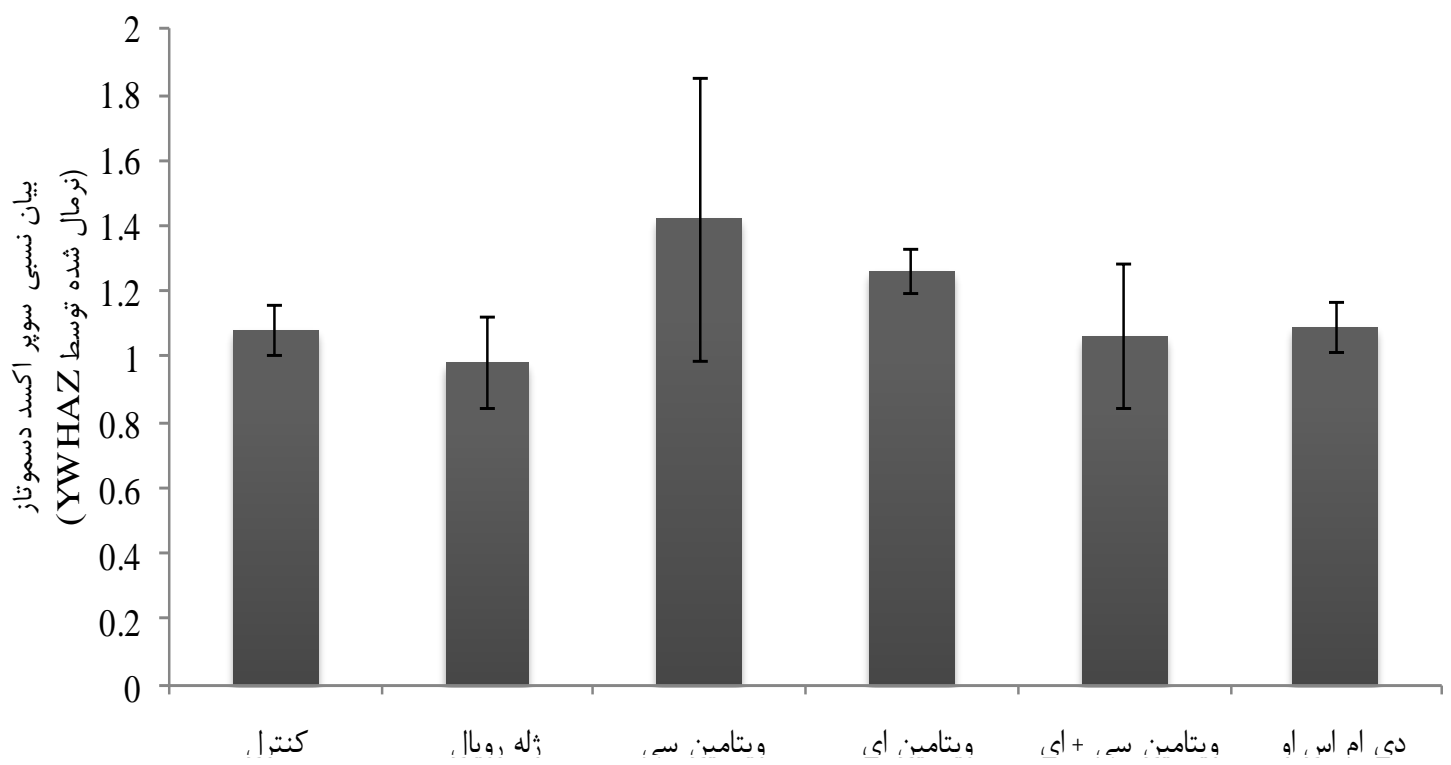

شكل سـ- تأثير افزودن ويتامينهاى E، C و زله رويال بر بيان نسبى ثن سويراكسيددسموتاز در اووسايت بز

Figure 3. Effect of vitamin E, C and royal jelly on superoxide dismutase relative gene expression in goat oocyte 


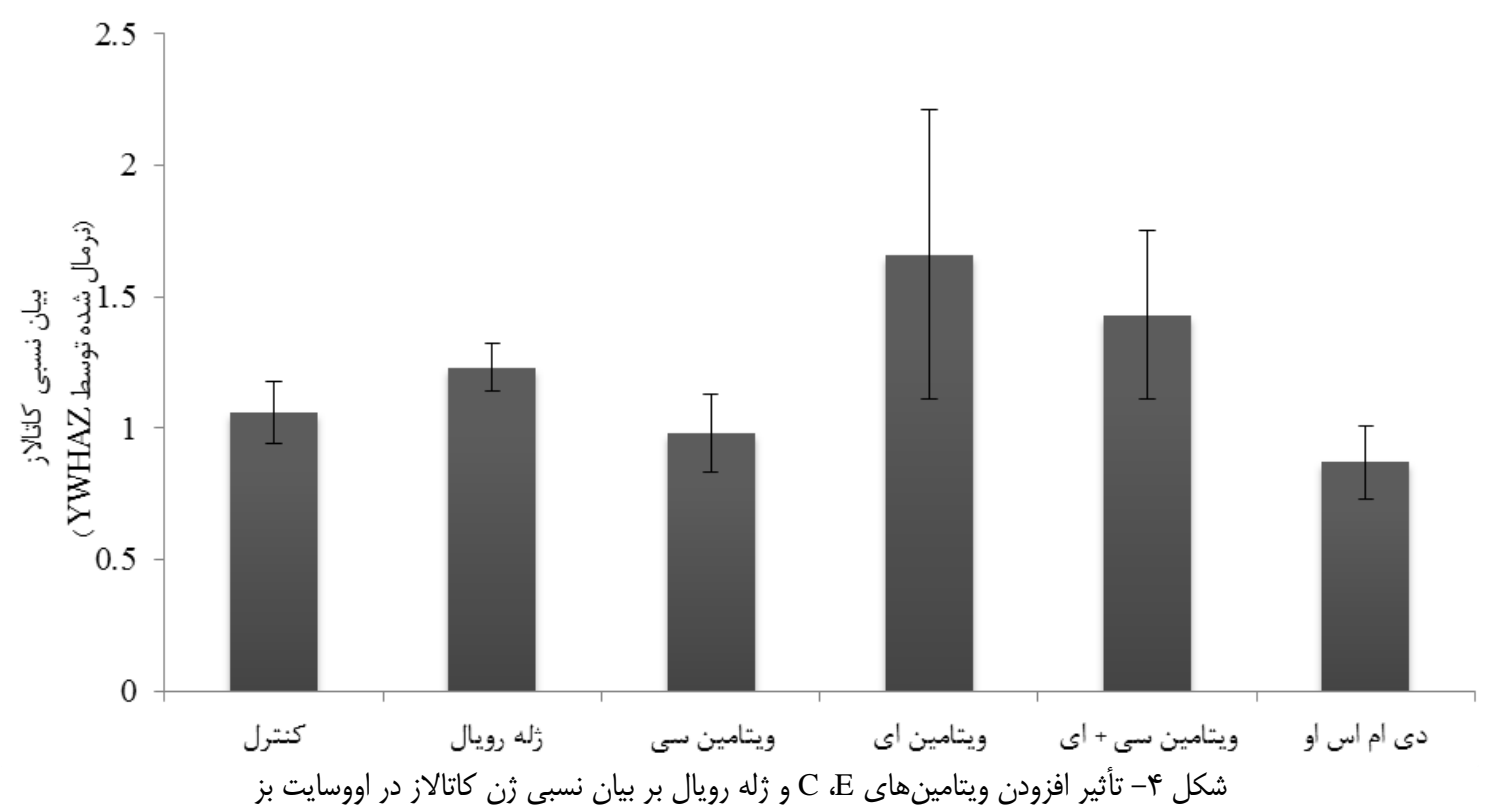

Figure 4. Effect of vitamin E, C and royal jelly on catalase relative gene expression in goat oocyte

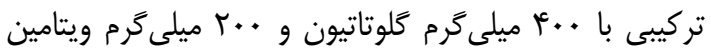

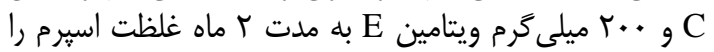

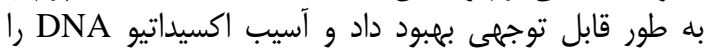

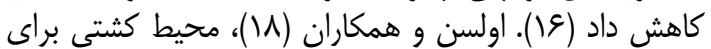

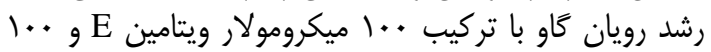

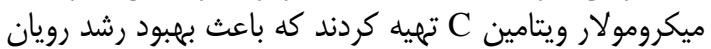

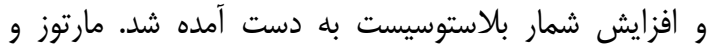

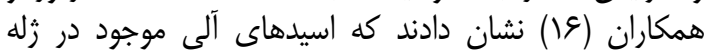

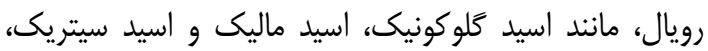

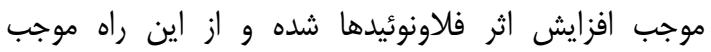

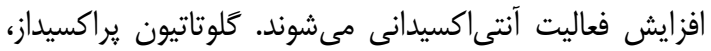

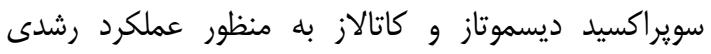

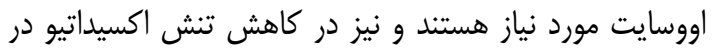

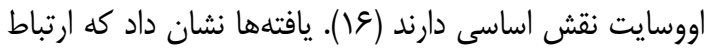

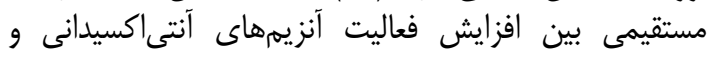
افزايش بلوغ سيتويلاسمى و هستهاى اووسايت، و در نتيجها بهبود نرخ بلاستوسيست، وجود دارد (أ) أران.

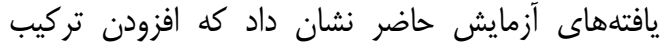

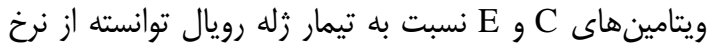

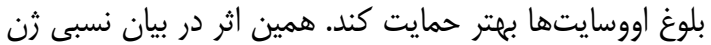

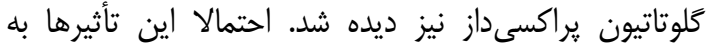

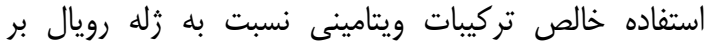

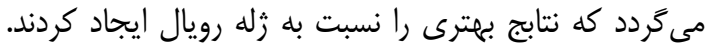

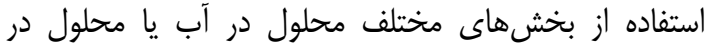

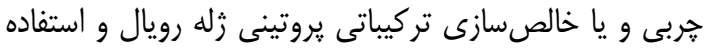

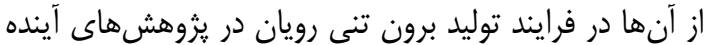

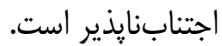

ويتامين E بهعنوان يك ماده محلول در خربى بوده كه كه

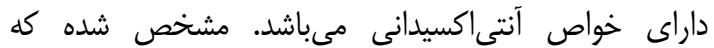

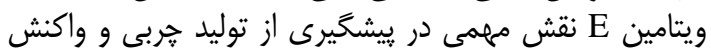

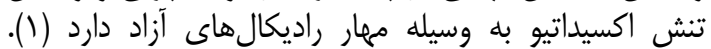

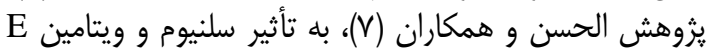

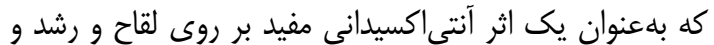

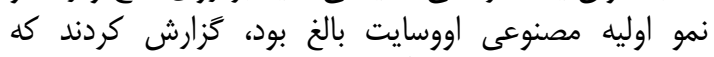

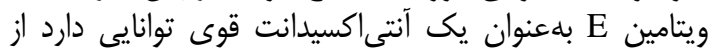

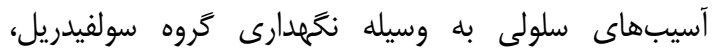

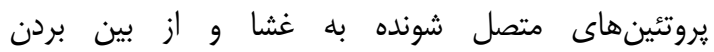

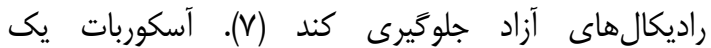

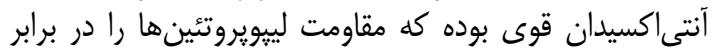

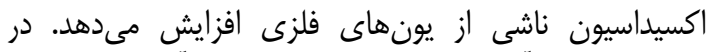

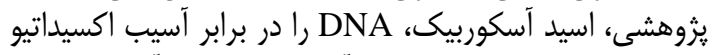

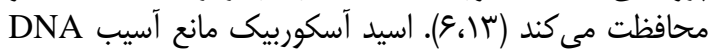

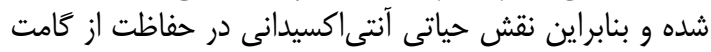

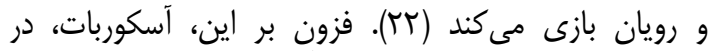

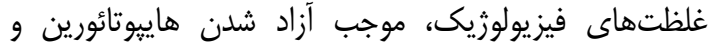

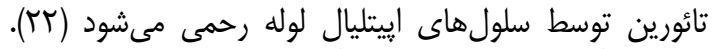

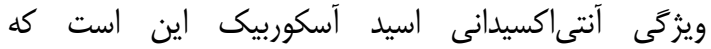

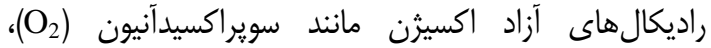
يراكسيد هيدروزن (إديكال

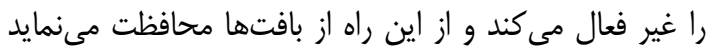

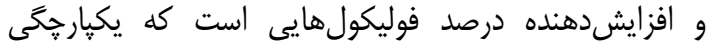

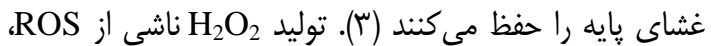

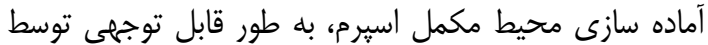

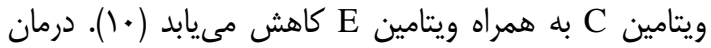


1. Asadi, E., M. Jahanshahi and M.J. Golalipour. 2012. Effect of Vitamin E on Oocytes Apoptosis in Nicotine-Treated Mice. Iranian Journal of Basic Medical Science, 3: 880-884.

2. Aslan, A., M. Cemek, M.E. Buyukokuroglu, K. Altunbas, O. Bas and Y. Yurumez. 2012. Royal jelly can diminish secondary neuronal damage after experimental spinal cord injury in rabbits. Food and Chemical Toxicology, 50: 2554-2559.

3. Barzegari Firozabadi, F. 2001. Effect of in vitro ascorbic acid and FSH on oocyte maturation and mouse follicles. Scientific Journal of Medical University shahid sadoghi yazd, 19: 586-597.

4. Cetica, P.L. and G. Pintos. 2002. Activity of key enzymes involved in glucose and triglyceride catabolism during bovine oocyte maturation in vitro. Reproduction, 124: 675-681.

5. Chow, C.K. 1991. Vitamin E and oxidative stress. Free Radical Biology and Medicine, 11: 215-232.

6. Donnelly, E.T., N. McClure and S.E. Lewis. 1999. The effect of ascorbate and alpha-tocopherol supplementation in vitro on DNA integrity and hydrogen peroxide-induced DNA damage in human spermatozoa. Mutagenesis, 14: 505-512.

7. Elhassan, Y.M. and R.W. Wright. 1995. The effect of selenium and vitamin E addition on cleavage rate of IVM/IVF bovine oocytes. Theriogenology, 43: 206-273.

8. Eshtiyaghi, M., H. Deldar and Z. Ansari pirsaraei. 2016. Royal jelly may improve the metabolism of glucose and redox state of ovin oocytes matured in vitro and emdryonic development following in vitro fertilization. Theriogenology, 86: 2210-2221.

9. Fraga, C.G., P.A. Motchnik and M.K. Shigenaga. 1991. Ascorbic acid protects against endogenous oxidative DNA damage in human sperm. Proceedings of the National Academy of Sciences, 88: $11003-11006$.

10. Guerin, P. and Y. Menezo. 1995. Hypotaurine and taurine in gamete and embryo environments: de novo synthesis via the cysteine sulfinic acid pathway in oviduct cells. Zygote, 3: 333-343.

11. Guo, H., Y. Kouzuma and M. Yonekura. 2008. Structures and properties of antioxidative peptides derived from royal jelly protein. Food Chemistry, 113: 238-245.

12. Jamnik, P., D. Goranovic and P. Raspor. 2007. Antioxidative action of royal jelly in the yeast cell. Experimental Gerontology, 42: 594-600.

13. Kamakura, M., M. Maebuchi., S. Ozasa and T. Ogawa. 2005. Influence of royal jelly on mouse hepatic gene expression and safety assessment with a DNA microarray. Nutritional Science and Vitaminology, 51: 148-155.

14. Karadeniz, A., N. Simsek, E. Karakus, S. Yildirim, A. Kara, I. Can, F. Kisa, H. Emre and M. Turkeli. 2011. Royal Jelly Modulates Oxidative Stress and Apoptosis in Liver and Kidneys of Rats Treated with Cisplatin. Oxidative Medicine and Cellular Longevity, $10 \mathrm{pp}$.

15. Kodama, H., R. Yamaguchi, J. Fukuda, H. Kasai and T. Tanaka. 1997. Increased oxidative deoxyribonucleic acid damage in the spermatozoa of infertile male patients. Fertility and Sterility, 68: 519-524.

16. Martos, M., Y.R. Navajas, J.F. Lopez and J.A.P. Alvarez. 2008. Functional properties of honey, propolis, and royal jelly. Journal of Food Science, 73: 117-124.

17. Mohammadi, S., H. Deldar and Z. Ansari pirsaraei. 2015. Effect of royal jelly on genes expression of antioxidant enzymes in in vitro maturation of goat oocytes. Master's thesis at Sari University of Agricultural Sciences and Natural Resources,

18. Olson, S.E. and G.E. Seidel. 2000. Culture of in vitro-produced bovine embryos with vitamin E improves development in vitro and after transfer to recipients. Biology of Reproduction, 62: 248-252.

19. Pavel, C.L., L.A.O. Marghitas, D.S. Bobis, A. Dezmirian, I. Sapcaliu and M.N. Madas. 2011. Biological activities of royal jelly. Animal Science and Biotechnology, 44: 108-118.

20. Pourmoradian, S., R. Mahdavi, M. Mobasseri, E. Faramarzi and M. Mobasseri. 2012. Effects of royal Jelly supplementation on body weight and dietary intake in type 2 diabetic females. Health Promotion Perspectives, 2: 231-235.

21. Shariatzade, S., M. Soleymani Mehranjani, A. Hamta and M. Ghandizade. 2012. Study the effect of vitamin $E$ on the structure and number of ovarian follicles during ovarian development in rats treated with sodium arsenite (sterological examination). Arak University of Medical Sciences Scientific Journal, 15: 54-64.

22. Valiollahpoor Amiri, M., H. Deldar and Z. Ansari Pirsaraei. 2016. Impact of supplementary royal jelly on in vitro maturation of sheep oocytes: genes involved in apoptpsis and embryonic development. System Biology in Reproductive Medicine, 62: 31-38. 


\title{
Effect of Royal Jelly, Vitamin C and Vitamin E on Genes Expression of Antioxidant Enzymes in in vitro Maturation of Goat Oocytes
}

\section{Saeed Heydari ${ }^{1}$, Hamid Deldar ${ }^{2}$ and Zarbakht Ansari Pirsaraei ${ }^{3}$}

\author{
1 and 3- Graduate Student and Associate Professor, Department of Animal Science, Sari Agricultural Sciences and \\ Naural Resources University \\ 2- Associate Professor, Department of Animal Science, Sari Agricultural Sciences and Naural Resources University \\ (Corresponding author: h.deldar@sanru.ac.ir)

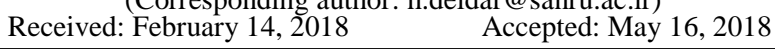

\begin{abstract}
The purpose of this study was to compare the antioxidant ability of royal jelly, vitamin $\mathrm{E}$ and vitamin $\mathrm{C}$ as additives to the maturation media on in vitro maturation of goat oocyte. To prepare goat oocytes, ovaries collected from local slaughterhouse were transported to the laboratory into the flask containing warm saline $\left(30-34^{\circ} \mathrm{C}\right)$ in less than an hour. Cumulus oocyte complexs were removed from small antral follicles $(2-6 \mathrm{~mm})$ with slicing method, and were transferred to IVM medium. Cumulus oocyte complexs were put in maturation medium for 24 hours, and were reached to metaphase meiosis II (nuclear maturation). This study was performed in a completely randomized design, with six treatments included control, $10 \mathrm{mg} / \mathrm{mL}$ royal jelly, $200 \mu \mathrm{M}$ of vitamin $\mathrm{E}, 100 \mu \mathrm{M}$ of vitamin $\mathrm{C}, 200 \mathrm{uM}$ of vitamin $\mathrm{E}+100 \mathrm{uM}$ of vitamin $\mathrm{C}$ and DMSO (as a solvent of vit $\mathrm{E}$ ) in ten replicates. The results of this study showed that addition of roval iellv. vitamin $\mathrm{E}$ and vitamin $\mathrm{C}$ to the maturation medium, increased in vitro maturation rate of goat oocvte in comnarison of control group. Supplementation of vitamin E, vitamin $\mathrm{C}$ and their combination significantly increased the oocyte meiotic maturation rate $(87.26 \% \pm 4.45)$ compared with the royal jelly $(76.26 \% \pm 2.55)$ and the control group $(71.1 \% \pm 1.26)$. Also, the addition of vitamin $\mathrm{E}+$ vitamin $\mathrm{C}$ combination was significantly increased relative gene expression of glutathione peroxidase but the superoxide dismutase and catalase were not affected by the treatment groups. In conclusion, the combination of vitamin $\mathrm{C}$ and vitamin $\mathrm{E}$ improved maturation rate and redox status of oocyte during in vitro maturation.
\end{abstract}

Keywords: Goat Oocytes, In vitro maturation, Royal Jelly, Vitamin E, Vitamin C 